\title{
PERSONA: a Psychometric Monitoring Instrument for High-stress Individuals
}

\author{
Nicholas H. Kirk* \\ Cambridge Humanae
}

\author{
Karmela Arbanasić \\ Cambridge Humanae
}

\begin{abstract}
Stress is considered to be among the leading causes of various psychopathological problems, whose outcomes are often difficult to foresee. In order to prevent consequent maladaptive behaviors, the present paper introduces a novel, three-phase architecture for analyzing the formation, handling and manifestation of stress in individuals. We specifically model the process of transforming stressful stimuli to cognitive schemas, and from these to subconscious defense mechanism manifestations. Such three-phase model has been implemented and validated via the PERSONA-80 test. Psychometric properties of PERSONA-80 have been evaluated on a sample of 83 individuals from general and clinical populations. The test showed good internal consistency and excellent predictive validity results, from which we derived the purified scale PERSONA-16. The potential of the developed instrument is the detection of the inability to produce mature defense mechanisms from an arbitrary state of mind, or the inability to preserve schemas when exposed to stressful conditions, useful in high-stress occupation screening and monitoring. To encourage widespread use and validation in different domains, we provide at the end of this text the complete PERSONA-16 instrument.
\end{abstract}

\section{Introduction}

Stress is considered to be one of the major causes of psychological problems to various extents, including psychopathological disorders, such as depression and anxiety, among many others. A lot of work has been done with the purpose of identifying the most common sources of stress and the most efficient coping strategies. For this, psychological instruments have been developed with the objective of diagnosing stress-induced psychopathologies. However, little work has been done with in measuring the process of stress formation, handling and manifestation. We would like, in this view, to introduce a novel functional, transactional perspective on psychometrics, focusing on the causal nature between stimuli, memories of the individual and behavioral response type.

More precisely, the work here presented describes a novel model for predicting stress-related psychopathological patterns, by introducing a three-phased transformation process of different stimuli, throughout an internal mental state, into an observational response. The present work empirically validates the ad-hoc created PERSONA test which implements such described three-phase model by relating stimuli optimality, cognitive schemas and coping strategies.

The three-phases measure different aspects of psychological functioning of the individual, which differ in level of stability over time. The first phase (henceforth PI) examines the need for various stimuli, which represent more stable personality traits, and which in certain situations lead to experiences of under-stimulation and over-stimulation. Such deviations from the optimal stimulation level are hereby defined as stressful experiences, which are being memorized and organized in the form of cognitive schemas. Cognitive schemas in the present framework represent the mental state of an individual which is the second phase of the model (henceforth referred to as PII). The third phase measures the output produced out of the current mental state in terms of emotional, cognitive and behavioral responses on the continuum of subconscious stress defense mechanism maturity, as intended in Vaillant's framework (Vaillant, Bond, \& Vaillant, 1986) (henceforth PIII). In this paper we show the validation of an ad-hoc constructed test comprising an implementation of the aforementioned PI, PII and PIII phases for a total of 80 items, which after sampling and empirical purification of scales, proved that the informativeness towards classifying certified clinical conditions is indeed present in all phases.

In our vision, phases are connected by different functions, parameters of which are hypothesized from empirical data. Such functions, which we define as conversion function and production function, cater for the aforementioned transformation between phases, by considering also nonlinearity. While these have been object of past qualitative analysis by the authors (Kirk \& Arbanasić, 2017b; Arbanasić \& Kirk, 2017), they will not be discussed further within this scope, as they represent an enhancement of the current model via the introduction on nonlinear estimation methods.

In the following chapters we will present a summary of the related work in stress assessment, to then introduce the developed PERSONA model in detail. The theoretical back-

*Please address any communication to nicholas.kirk@tum.de 


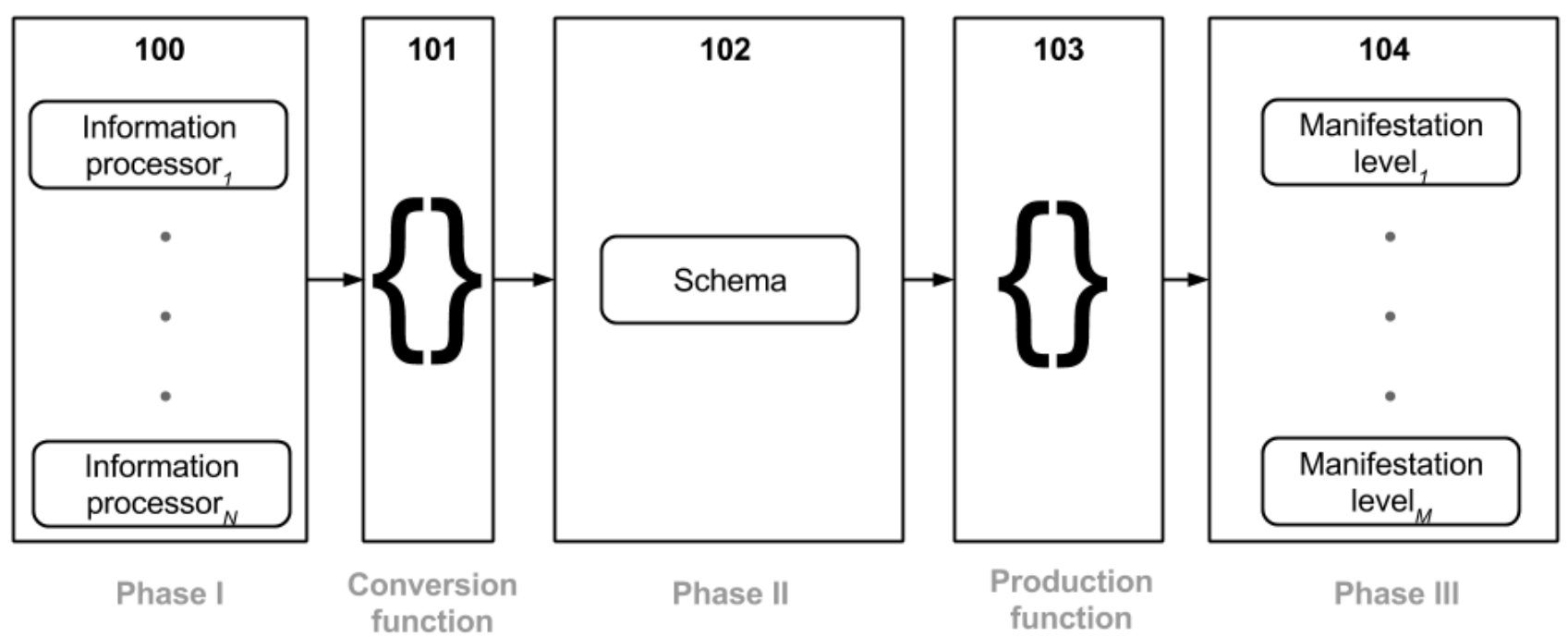

Figure 1. Schematic diagram illustrating the three-phase architecture of the model, presenting a transformational process of input to output throughout three sequential phases and two functions inbetween which connect them

ground of this will be presented, followed by an empirical validation of such three-phase architecture with the PERSONA test. Methodological limitations as well as implications for future work will be discussed in the end.

\section{Related Work}

In this chapter, we will present the most relevant findings in the field of stress-coping and its relations to cognitive schemas and stimuli optimality. We will compare some of the existing approaches for stress-coping assessment to our model, and emphasize the innovative aspects of the here introduced PERSONA test. Previous work in the field of stress-coping analysis identifies two different approaches. The first approach focuses on the efficiency of various coping strategies, which involve conscious, purposeful effort aimed at solving the problem situation (Cramer, 1998). One of the most known measures implementing this approach is the COPING INVENTORY FOR STRESSFUl Situations (Endler, Parker, de Ridder, \& van Heck, 2004), which divides strategies in task-oriented coping, emotion-oriented coping, avoidance-oriented coping, distraction and social diversion. Another widely used test is the COPE InVENTORY (Carver, Scheier, \& Weintraub, 1989), which measures problem-focused, emotion-focused and "less useful" strategies. The second approach focuses on defense mechanisms, which occur without conscious effort and intentionality (Cramer, 1998). Vaillant defined them as innate, involuntary regulatory processes which enables individuals to reduce cognitive dissonance and to minimize sudden changes from the environment by modifying the perceptions of these events (Vaillant, 1994). Vaillant and Bond created the hierarchy of defense mechanisms (Vaillant et al., 1986), upon which Bond developed the Defense Style QuestionNAIRE (DSQ) (Andrews, Singh, \& Bond, 1993). DSQ ranks defense mechanisms on a developmental continuum, from maladaptive, through image-distorting and self-sacrificing to adaptive. Maladaptive mechanisms correlated with symptoms of personality disorders, depression and anxiety disorders (Bond, 2004).

Conversely in our view, stress defense mechanisms are a direct consequence of under- and over-stimulation, meaning that deviations from the desired level of stimulation of an individual cause stressful experiences, which activate given defense strategies in individuals. Previous work performed by the authors provided the initial rationale and qualitative analysis of the necessary latent variables and their relations (Kirk \& Arbanasić, 2017b, 2017a; Arbanasić \& Kirk, 2017).

The relationship between the type of stimuli and stress level has been explored in some of the previous work in this field. Studies examining stress sources in police officers found that officers with high-workload were stressed above all by work tasks, while officers with low workload were stressed more by social aspects within and outside the workplace (Stotland $\&$ Pendleton, 1989). This implies that different kind of stimuli can cause stress in different contexts. The amount of stress, however, depends on the individual's optimal stimulation level (OSL) (Hebb, 1955). Optimal stimulation theory, introduced by Hebb (Hebb, 1955) and Leuba (Leuba, 1955), states that each individual has a certain preferred level of arousal, which he/she will try to maintain at the optimum level (Raju, 1980). This stimulation can come from the internal states or the environment (Steenkamp \& Baumgartner, 1992) and can be of cognitive or sensorial nature (Zuckerman, 1994). Pearson went a step further, and classified individuals according to a preferred type of stimuli, 
based on the source (external vs internal) and nature (sensorial vs cognitive) of stimuli (Pearson, 1971). Stimuli preferences were found to be related to coping strategies as well; both external and internal sensation were negatively related to repression, while internal cognitive was positively related to sensitization. In research regarding workload and stress in police officers, the low quality of relationships induced stress in low-workload officers by decreasing their selfesteem (Stotland \& Pendleton, 1989), which implies that bad social stimuli lead to stress by impacting our self-schema. Furthermore, it has been shown that people who have been exposed to stressful experiences perceive themselves as less worthy and the world as less benevolent (Janoff-Bulman, 1989; Dekel, Solomon, Elklit, \& Ginzburg, 2004). We can therefore conclude that stressful stimuli impact our cognitive schemas about self and the world. According to the underlying theory of Brief Core Schema Scale (BCSS) (Fowler et al., 2006), the evaluations of the individual regarding the self and the world determine his/her responses in stressful situations and adaptation to the social world. Extreme negative evaluations of the self and the world were found to be associated with depression and PTSD (Fowler et al., 2006). This leads to the conclusion that cognitive schemas about self and the world can influence one's coping responses, concept which we leverage within this scope. The above described findings provided theoretical rationale for development of the model in which we bridge stimuli optimality, cognitive schemas and defense mechanisms. The model was inspired by the above mentioned Bond's and Vaillant's hierarchy of defense mechanisms on the continuum of maturity (Vaillant et al., 1986). We also hereby rely on Kramer's statement that defense mechanisms function to change the internal psychological state, but may have no effect on external reality, which may result in reality distortion (Cramer, 1998). We leverage this fact and further this concept in considering reality distortion as a spectrum of cognitive schema distortion. At the moment there are no available psychological instruments which measure the process of elaborating stressful stimuli throughout a mental state which then consider the production of coping responses. We therefore focused on the construction of a stress-oriented psychometric test which would relate stimuli optimality, cognitive schemas and coping maturity in order to provide an accurate measure for process of generating and handling stressful stimuli, rather than a set of traits or responses.

\section{Model}

As stated above, the present model describes how the incoming stimuli are being processed throughout a mental state and transformed into coping responses. The first phase acts as an input, and refers to individual's optimal level of stimulation from different sources of stimuli: Both redundancy and lack of stimuli create certain stressful experiences which are elaborated and memorized. In the second phase, memories are organized into cognitive schemas of oneself and of the world, which represent the current mental state of the individual. In the third phase, the current mental state produces a response to the stressful experience, in form of coping strategies, which act as an output of the cognitive process. The now described architecture is illustrated in Figure 1.

Phase I: Need for stimuli. The first phase examines the individual's need for various stimuli which differ by their sources and by their nature. This phase represents an aspect of personality, which is unique for each individual, which is shaped by genetic, environmental or sociogenic factors and which is most stable over time. In the context of the present model, stimuli act as input from the environment which is being received and processed according to needs of the individual, thereafter memorized and organized in his/her cognitive schemas. The observations from this phase differentiate individuals by their optimal level of stimulation and their preferences for certain stimuli. We assume that people differ not only by the desired level of stimulation, but also by the preferred sources of stimuli. We hereby refer to Pearson's categorization (Pearson, 1970), which divides needs for experiences into four preferences, based on sources of stimuli, depending on whether they are self-generated (internal) or environmental (external), and whether they are sensorial or cognitive by nature. Since it has been shown that also the social aspect plays role in recall of autobiographical memories (i.e. extroverted individuals were faster to recall pleasant experiences (Lishman, 1974) and recalled more pleasant memories than introverts (Mayo, 1983)), we divided each category into the orientation towards the self and orientation towards other people.

Phase II: Cognitive schemas. The second phase examines the way that perceptions of over-stimulation and understimulation create autobiographical memories and how these memories organize into cognitive schemas of one's self (henceforth self-schema) and of world (henceforth worldschema). The combination of these represents the current mental state of an individual, shaped by environmental factors and psychosocial situations, and therefore it is the least stable latent trait over time we consider. In the context of the present model, a mental state represents a container of created memories from which we determine the individual's reactions in stressful situations. The observations from this phase are based on memory retrieval, and differentiate individuals by the way how they memorize and recall certain experiences of stimulation in terms of positivity and importance. We hereby refer to the underlying theory of the Brief Core Schema Scale (Fowler et al., 2006), which assesses positive and negative evaluations of the self and of others. However, it was found that the impact of an individual's selfperceptions on his/her self-schema is mediated by the relative importance he/she attributes to them (Pelham \& Swann, 
1989). The present model therefore assumes that individuals who give less importance to positive memories and more importance to negative ones are more likely to generate a negative internal state, which will most likely lead to the adaptation towards immature coping behaviors in stressful situations. Meanwhile, individuals who consider their negative memories less important, thus minimizing their impact and focusing on positive memories, will more easily create a positive internal state and apply mature coping mechanisms under stressful circumstances.

Phase III: Coping strategies. The third phase examines the maturity of an individual's emotional, cognitive and behavioral responses to perceived stressful experiences. In the context of the present model, coping mechanisms act as an output produced as a result of the current mental state. The observations from this phase differentiate individuals by their capability of mature stress handling, without exhibiting problematic or dangerous behavior and exposing themselves, or others, to danger. We hereby refer to Bond's and Vaillant's hierarchical model (Vaillant et al., 1986) whose levels of coping maturity correlated strongly with certain psychopathological disorders. It has been found that usage of less mature mechanisms was related to different psychological disorders, such as borderline personality disorder (Bond, Paris, \& Zweig-Frank, 1994) and anxious disorders (Andrews, Pollock, \& Stewart, 1989). In the present model we define maturity as the extent of reality distortion, where we assume that lower levels of adaptive functioning include major cognitive biases of reality perception. In particular, this model aims to differentiate individuals by the extent to which the individual distorts world-image and self-image when subjected to stressful experiences, in order to capture the degree of misalignment between the individual and the world around him/her. We hypothesize that individuals who are capable of adjusting self-image to the world-image will show more adaptive behavior, while individuals who tend to adjust the world-image to the self-image are more likely to develop maladaptive behavior and psychological disorders.

\section{Implementation}

The above presented model provided the theoretical rationale for the construction of the PERSONA questionnaire. PERSONA questionnaire is a psychological test which implements the earlier described three-phase architecture to measure a transformation process of stimuli under- and overstimulation into a mental state, later manifested as coping strategies. The test is an 80-item self-report based measure. Items are scored using a 7-Likert scale, with responses ranging from 1 ("completely disagree") to 7 ("completely agree"). In order to monitor demographic variables, the participant is asked to indicate gender, age, marital status, educational status and employment status before replying to the items. Since the test evaluates stress/coping strategies, the partici-
Table 1

Enumeration and explanation of the implemented "Need for stimuli" dimensions

\begin{tabular}{|c|c|}
\hline Need for stimuli & Explanation \\
\hline External cognitive - self oriented & $\begin{array}{l}\text { Need for receiving cognitive } \\
\text { information }\end{array}$ \\
\hline External cognitive - oriented to others & $\begin{array}{l}\text { Need for exchanging cognitive } \\
\text { information with others }\end{array}$ \\
\hline External sensorial - self oriented & Need for engaging in physical activities \\
\hline External sensorial - oriented to others & $\begin{array}{l}\text { Need for receiving affection from } \\
\text { others }\end{array}$ \\
\hline Internal cognitive - self oriented & $\begin{array}{l}\text { Need for reflection about things, people } \\
\text { and events }\end{array}$ \\
\hline Internal cognitive - oriented to others & $\begin{array}{l}\text { Need for exchanging thoughts and } \\
\text { ideas with others }\end{array}$ \\
\hline Internal sensorial - self oriented & Need for experiencing emotions \\
\hline Internal sensorial - oriented to others & Need for sharing emotions with others \\
\hline
\end{tabular}

pant is also asked to indicate the level of stress he/she experienced in the last six months; from 1 (very low) to 5 (very high). The construction in terms of item composition of each phase is described in the following paragraphs and illustrated in Figure 2.

PHASE I measures individual's optimal level of stimulation and his/her preferences for a given type of stimuli, relying on Pearson's categorization (Pearson, 1970), differentiating by the source of stimuli (internal vs external) and by the nature of stimuli (sensorial vs cognitive). Stimuli are therefore divided into four categories; external sensorial, external cognitive, internal sensorial and internal cognitive; and we further subdivided each category into self-oriented and people-oriented. Items were formed with a purpose of examining the extent to which a person feels the need for such kind of experiences. The total number of items in this phase is 8 , one for each subscale, and they begin with "I need...". Each category is explained in Table 1.

PHASE II attempts to maps under- and over-stimulation from different stimuli by means of memory retrieval, on the continuum of positivity-negativity and importanceunimportance. Memories of past experiences are divided on the basis of the same categorization as in the PHASE I. Importance of memories is evaluated by items which assess how often does a person think about certain experiences, while positivity of memories is evaluated by items which assess how positive does a person feel when he/she thinks about certain experiences. Since the total number of items for this dimension is 32 and the items are similar in form, we decided to reverse half of the items by means of preventing response bias. Therefore, 8 items measuring importance begin with "I often think of..." while other 8 begin with "I rarely think of...". In the same way, 8 items measuring positivity begin with "I feel positive when I think of...", while other 8 begin with "I feel negative when I think of...".

PHASE III measures responses of the perceived stressful experiences on the maturity continuum, where the maturity is defined as the extent of a reality distortion, i.e. distortion of the world-image and the self-image in stressful situations on 


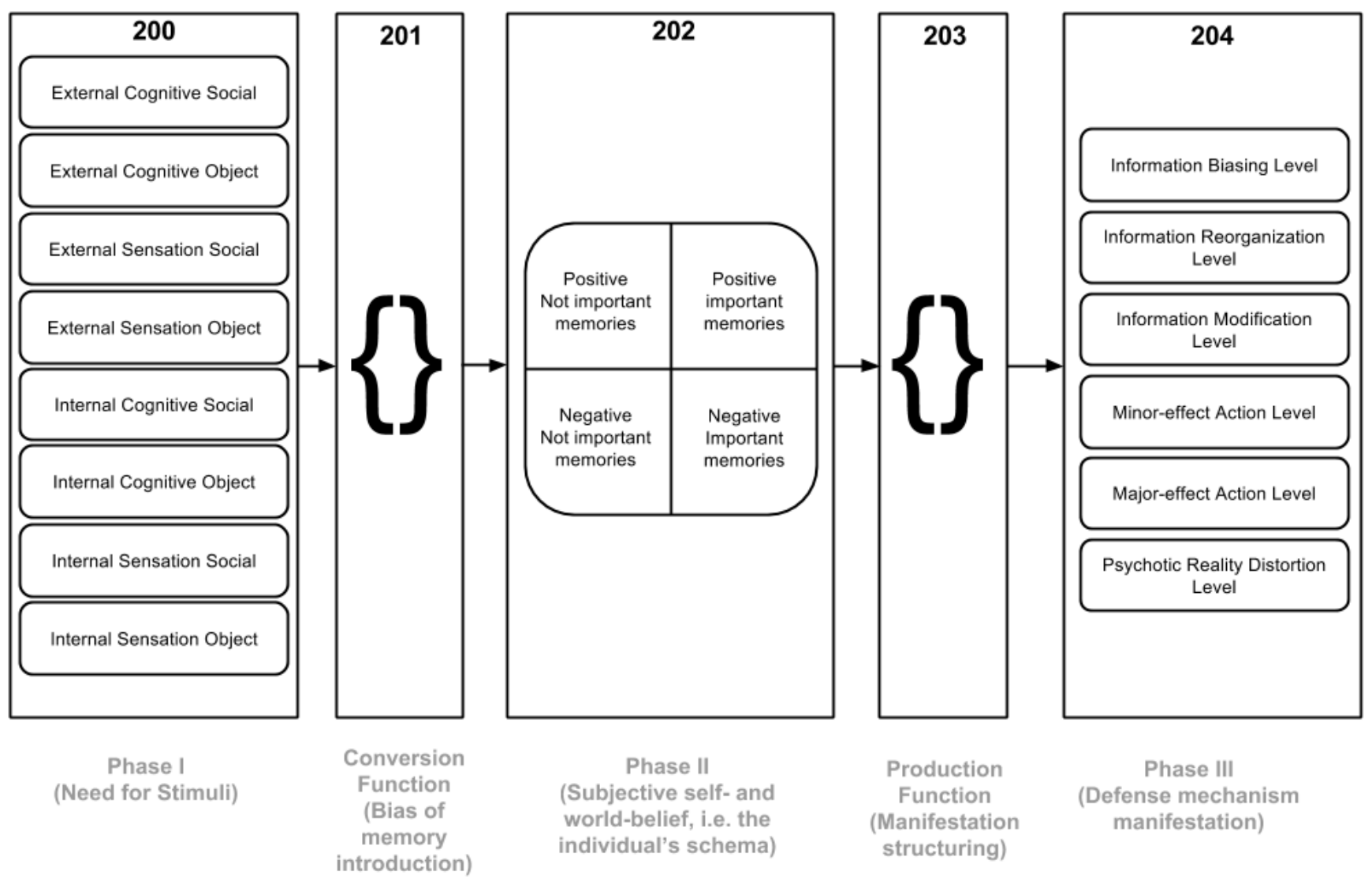

Figure 2. Schematic diagram illustrating the psychometric implementation of the model, presenting psychological variables measured in three phases

an emotional, cognitive and behavioral level. Higher levels of the continuum contain a lower extent of reality distortion which mostly includes cognitive patterns, while lower levels of maturity contain higher extent of reality distortion, based on more emotional and behavioral patterns. We divided the mechanisms in 6 levels, where each level contains a certain number of individual defense mechanisms. The total number of items in this phase is 40 , whereas the lowest dimension contains 15 items and the higher dimensions contain 5 items each. The lowest dimension has been more saturated during the design phase in order to capture maladaptive coping patterns more effectively. Definitions of individual levels are provided in Table 2.

\section{Evaluation}

\section{Data collection}

From June 2017 to November 2017, PERSONA was administered, with a high degree of diligence, to 83 individuals. Sampling was conducted within the general and clinical population. The sample from general population comprises 67 individuals without certified psychiatric diagnosis and who weren't receiving psychiatric or psychological treatment at
Table 2

Levels of reality distortion hypothesized for Phase III

\begin{tabular}{|c|c|}
\hline Coping level & Definition \\
\hline Information bias & $\begin{array}{l}\text { Bias in attributing importance to certain kind of } \\
\text { information, with the objective of protecting the } \\
\text { self-schema }\end{array}$ \\
\hline Information reorganization & $\begin{array}{l}\text { Excessive rationalization and avoidance of } \\
\text { emotional reactions, with the objective of } \\
\text { protecting the self-schema }\end{array}$ \\
\hline Information modification & $\begin{array}{l}\text { Deliberate modification of reasoning or behavior } \\
\text { with the objective of protecting the self-schema }\end{array}$ \\
\hline Indirect action & $\begin{array}{l}\text { Passive expression of negative emotions toward } \\
\text { others with the objective of protecting the } \\
\text { self-schema }\end{array}$ \\
\hline Direct action & $\begin{array}{l}\text { Active expression of negative emotions toward } \\
\text { others with the objective of protecting the } \\
\text { self-schema }\end{array}$ \\
\hline Reality distortion & $\begin{array}{l}\text { Intentional modification of world-perception with } \\
\text { the objective of protecting the self-schema }\end{array}$ \\
\hline
\end{tabular}

the moment of testing. Conversely, the sample from the clinical population comprises 16 clinical patients from Santa Chiara hospital in Pisa (Italy) with certified psychiatric diagnosis, which was provided by a psychiatrist or psychologist and/or from the patient's medical chart. Certified diagnoses included gambling disorder, major depressive disorder, panic disorder and borderline personality disorder. Both samples 
Table 3

Results of the Confirmatory Factor Analysis (CFA) for Phase I of PERSONA-80, displaying a 2-factor solution

\begin{tabular}{llc}
\hline & \multicolumn{2}{c}{ Component } \\
\hline Items & \multicolumn{1}{c}{$\mathbf{1}$} & $\mathbf{2}$ \\
PI.ES.P & $\mathbf{0 . 7 3 5}$ & -0.199 \\
PI.IS.P & $\mathbf{0 . 7 0 2}$ & 0.224 \\
PI.EC.O & $\mathbf{0 . 6 2 7}$ & 0.253 \\
PI.ES.O & $\mathbf{0 . 6 1 8}$ & 0.306 \\
PI.IS.O & $\mathbf{0 . 6 0 9}$ & 0.272 \\
PI.IC.P & 0.23 & $\mathbf{0 . 8 0 6}$ \\
PI.IC.O & 0.201 & $\mathbf{0 . 6 8 5}$ \\
PI.EC.P & 0.591 & $\mathbf{0 . 6 6 4}$ \\
\hline
\end{tabular}

Table 4

Results of the Confirmatory Factor Analysis (CFA) for Phase II of PERSONA-80, displaying a 2-factor solution

\begin{tabular}{lll}
\hline & \multicolumn{2}{c}{ Component } \\
\hline Items & $\mathbf{1}$ & \multicolumn{1}{c}{$\mathbf{2}$} \\
PII.OP.IC.P & $\mathbf{0 . 7 8 6}$ & 0.174 \\
PII.SP.IC.O & $\mathbf{0 . 7 6 1}$ & 0.093 \\
PII.OP.EC.O & $\mathbf{0 . 7 3 6}$ & 0.229 \\
PII.SP.EC.P & $\mathbf{0 . 7 2 4}$ & 0.273 \\
PII.OP.EC.P & $\mathbf{0 . 6 4 8}$ & 0.155 \\
PII.SP.IS.O & $\mathbf{0 . 6 4 6}$ & 0.301 \\
PII.OP.IS.P & $\mathbf{0 . 5 7 3}$ & 0.305 \\
PII.SP.ES.O & $\mathbf{0 . 5 4 9}$ & 0.049 \\
PII.OI.EC.O & $\mathbf{0 . 5 4 7}$ & 0.29 \\
PII.SP.IS.P & $\mathbf{0 . 5 4 4}$ & 0.253 \\
PII.OP.ES.O & $\mathbf{0 . 5 4 4}$ & 0.091 \\
PII.SP.EC.O & $\mathbf{0 . 5 3 4}$ & -0.068 \\
PII.SP.ES.P & $\mathbf{0 . 5 3 1}$ & 0.128 \\
PII.SP.IC.P & $\mathbf{0 . 5 0 3}$ & -0.055 \\
PII.OP.IC.O & $\mathbf{0 . 4 9 8}$ & 0.176 \\
PII.OP.IS.O & $\mathbf{0 . 4 6 3}$ & 0.048 \\
PII.OI.EC.P & $\mathbf{0 . 4 0 9}$ & 0.268 \\
PII.SI.IS.P & 0.048 & $\mathbf{0 . 6 9 8}$ \\
PII.SI.IC.P & -0.119 & $\mathbf{0 . 6 9 4}$ \\
PII.OI.IC.P & 0.288 & $\mathbf{0 . 6 3 7}$ \\
PII.SI.ES.P & 0.199 & $\mathbf{0 . 6 2 2}$ \\
PII.OI.IS.P & 0.437 & $\mathbf{0 . 5 9 3}$ \\
PII.SI.IS.O & 0.035 & $\mathbf{0 . 5 8 6}$ \\
PII.OI.IS.O & -0.079 & $\mathbf{0 . 5 6 4}$ \\
PII.OI.ES.P & 0.191 & $\mathbf{0 . 5 5}$ \\
PII.SI.IC.O & 0.246 & $\mathbf{0 . 5 4 6}$ \\
PII.OI.IC.O & 0.399 & $\mathbf{0 . 4 9 3}$ \\
PII.SI.EC.P & 0.273 & $\mathbf{0 . 4 3 2}$ \\
PII.SI.ES.O & 0.122 & $\mathbf{0 . 4 0 8}$ \\
PII.OI.ES.O & 0.187 & $\mathbf{0 . 4 0 1}$ \\
PII.SI.EC.O & 0.022 & $\mathbf{0 . 3 4 2}$ \\
PII.OP.ES.P & 0.185 & $\mathbf{0 . 2 5}$ \\
\hline & &
\end{tabular}

were voluntary and testing was conducted in controlled conditions. Participants were welcomed in the room with appropriate lighting and temperature, without noise and in the early afternoon, to avoid biological factors such as hunger and tiredness. They were asked to put away their phones, and to go to the toilet before the testing if necessary, in order not to get distracted during the testing. It is important to note that the general sample was tested with the English version of PERSONA, while the clinical sample was tested with the Italian version of PERSONA. Although this could bring a certain bias into the interpretation results and reduce the reliability of the test, as we will demonstrate herein, internal consistency remained sufficiently high in our evaluation. The two samples were merged into one dataset and validation
Table 5

Results of the Confirmatory Factor Analysis (CFA) for Phase III of PERSONA-80, displaying a 2-factor solution

\begin{tabular}{lll}
\hline & \multicolumn{1}{c}{ Component } \\
\hline \multicolumn{1}{c}{ Items } & \multicolumn{1}{c}{$\mathbf{1}$} & \multicolumn{1}{c}{$\mathbf{2}$} \\
PIII.IR.5 & $\mathbf{0 . 6 5 9}$ & -0.19 \\
PIII.RD.5 & $\mathbf{0 . 6 1 6}$ & 0.002 \\
PIII.IR.4 & $\mathbf{0 . 5 9 2}$ & -0.324 \\
PIII.IB.2 & $\mathbf{0 . 5 8 4}$ & -0.245 \\
PIII.IR.2 & $\mathbf{0 . 5 7 3}$ & -0.016 \\
PIII.IB.5 & $\mathbf{0 . 5 4 6}$ & -0.046 \\
PIII.DA.5 & $\mathbf{0 . 5 3 7}$ & 0.118 \\
PIII.RD.11 & $\mathbf{0 . 5 1 4}$ & -0.272 \\
PIII.RD.4 & $\mathbf{0 . 5 0 5}$ & -0.136 \\
PIII.RD.15 & $\mathbf{0 . 4 7 7}$ & -0.3 \\
PIII.RD.6 & $\mathbf{0 . 4 3}$ & 0.144 \\
PIII.RD.8 & $\mathbf{- 0 . 4 0 6}$ & -0.079 \\
PIII.RD.10 & $\mathbf{0 . 3 9 7}$ & -0.194 \\
PIII.IM.5 & $\mathbf{0 . 3 6 9}$ & 0.347 \\
PIII.RD.7 & $\mathbf{- 0 . 3 6}$ & 0.335 \\
PIII.IB.1 & $\mathbf{0 . 3 2 2}$ & -0.231 \\
PIII.IM.4 & $\mathbf{0 . 3 2 1}$ & 0.11 \\
PIII.RD.9 & $\mathbf{0 . 3 2}$ & -0.056 \\
PIII.DA.3 & $\mathbf{0 . 3 0 2}$ & -0.064 \\
PIII.RD.3 3 & $\mathbf{0 . 2 6 4}$ & 0.167 \\
PIII.RD.12 & $\mathbf{0 . 2 5 6}$ & -0.006 \\
PIII.IM.2 & $\mathbf{0 . 2 2 9}$ & 0.009 \\
PIII.IM.3 & -0.203 & $\mathbf{0 . 6 7}$ \\
PIII.RD.13 & -0.091 & $\mathbf{0 . 6 5}$ \\
PIII.RD.1 & -0.081 & $\mathbf{0 . 6 0 5}$ \\
PIII.DA.2 & -0.018 & $\mathbf{0 . 5 8 4}$ \\
PIII.IA.3 & -0.048 & $\mathbf{0 . 5 5 1}$ \\
PIII.RD.2 & 0.127 & $\mathbf{0 . 5 1 7}$ \\
PIII.IA.1 & -0.251 & $\mathbf{0 . 4 7 4}$ \\
PIII.IM.1 & -0.093 & $\mathbf{0 . 4 2 5}$ \\
PIII.IA.4 & 0.131 & $\mathbf{0 . 4 0 3}$ \\
PIII.IA.5 & 0.253 & $\mathbf{0 . 3 9 3}$ \\
PIII.DA.1 & -0.301 & $\mathbf{0 . 3 8 4}$ \\
PIII.IB.4 & -0.051 & $\mathbf{0 . 3 3 5}$ \\
PIII.DA.4 & -0.188 & $\mathbf{0 . 3 2 2}$ \\
PIII.IR.3 & 0.296 & $\mathbf{0 . 3 1 5}$ \\
PIII.IA.2 & 0.11 & $\mathbf{0 . 2 8 3}$ \\
PIII.IB.3 & -0.197 & $\mathbf{0 . 2 0 2}$ \\
PIII.RD.14 & 0.102 & $\mathbf{- 0 . 1 9 5}$ \\
PIII.IR.1 & 0.024 & $\mathbf{- 0 . 1 3 7}$ \\
\hline & &
\end{tabular}

analysis was performed on all 83 cases. Each case consists of 86 observations. 6 observations include demographic informations, while 80 observations include self-reported level of agreement with claims regarding the measured constructs. Dataset was balanced with regards to gender, with 54.2percent of male participants. With regards to age balance, 79.5-percent of participants belonged to age group from 19 to 40 years.

\section{Data analysis}

In order to evaluate the above described model, we performed two series of experiments. In the first group of experiments we validate the PERSONA test, called PERSONA80 by analyzing its psychometric properties. In particular we provide results regarding reliability and predictive validity of the measured constructs and conclude with the extraction of items with maximum predictive strength towards psychopathological condition detection with which we construct the purified PERSONA-16 scale. In the second group of experiments we validate psychometric properties of 
PERSONA-16 scale.

Stage I: Psychometric properties of PERSONA80. In stage I we assessed psychometric properties of PERSONA- 80 by examining normality of distributions, as well as reliability intended as internal consistency and validity, i.e. in terms of construct validity and predictive validity.

Table 6 displays main variance and distribution statistics. A low variance coefficient for all three-phases indicates low level of dispersion in their distributions. Normality of distribution was assessed with Kolmogorov-Smirnov test and Shapiro-Wilk test, whose results are presented in Table 7. Only PHASE I showed significant deviation from normal distribution, by showing leptokurtic distribution, as it can be seen from Table 6 . We attribute this deviation from normality to low number of items in this scale.

Cronbach alpha was computed in order to assess internal consistency of each phase. As presented in Table 8, PHASE I and PHASE II showed strong internal consistency, both exceeding the recommended 0.7 cutoff (Nunally \& Bernstein, 1994), whereas PHASE I had good consistency (0.74), and PHASE II exhibited very good consistency (0.88). PHASE III showed moderate to good internal consistency, an remained at 0.68 , which is still acceptable given the heterogeneous set of behaviors measured by this scale. Details about the itemlevel statistics are provides in the Appendix, in Table 16.

Construct validity was estimated with Confirmatory Factor Analysis (CFA), in order to evaluate hypotheses about the model structure. Oblimin rotation was applied since the factors are assumed to be correlated in this model. 15 out of 80 items haven't met the standard criteria of 0.30 (Nunally $\&$ Bernstein, 1994). Three factors, presented in Table 14, explained 29.5-percent of variance: the first factor accounts for 12.43-percent of variance and contains 38 items, equally distributed among all three-phases, including majority of items from PHASE I; the second factor accounts for additional 5.96-percent of variance and contains 20 items, including a portion of items from PHASE II from the subscale IMPORTANCE and a portion of items from PHASE III; the third factor explains an additional 5.2-percent of variance and contains 22 items, including a portion of items from PHASE II, from the subscale Positivity and portion of items from PHASE III.

Confirmatory Factor Analysis (CFA) was performed on the scale-level as well, in order to identify subscales for each phase of the model. 2 factors were extracted for every phase: factor loadings from PHASE I, called Needs, imply division on Cognitive and Sensorial needs, which should become clearer with the increase of data entries; factor loadings from PHASE II, called Cognitive schemas, show sufficiently clear division on the Positivity and the Importance dimensions of memories; factor loadings from PHASE III, called Coping strategies, show as well a sufficiently clear division on Inneroriented coping and Outer-oriented coping. Results for factor solutions for each phase can be found in Table 3, 4 and 5.

Predictive validity was assessed as a function of accuracy in discriminating between classes. As a result, a classification rate of 97-percent accuracy was obtained and 16 items with most predictive strength were extracted and computed into a final, purified scale, named PERSONA-16. Dimensions are presented in the Table 9 above, and the items of which are provided in detail in Table 17. In the next stage, psychometric properties of the purified scale will be analyzed.

Stage II: Psychometric properties of PERSONA-16. Since this scale has been constructed for the scope of predictive validity out of extracted items of a larger subset, we can expect certain psychometric properties to be weaker than the original PERSONA-80. Hence, in Table 10 one can see that PERSONA-16 shows leptokurtic and negatively skewed distribution, while values of Kolmogorov-Smirnov and Shapiro-Wilk test in Table 11 confirm that it significantly deviates from normal distribution. We attribute this lack of convergence to the restricted dimensionality of the samples.

However, internal consistency of PERSONA-16, presented in Table 12, remains strong, with Cronbach Alpha of 0.72 . Although this scale is constructed from items from all three-phases, this result shows that they are sufficiently intercorrelated, which is as expected because the extracted items predict the same class.

Since PERSONA-16 has been empirically constructed, rather than based on theoretical hypotheses, factor structure was assessed with Exploratory Factor Analysis (EFA) with oblimin rotation, which allows correlations between factors. All items met the standard criteria of 0.30 (Nunally \& Bernstein, 1994) and were retained. On the basis of Kaiser value (Kaiser, 1960) and the scree test (Cattell, 1966), 5 factors, which together explain 63.64-percent of variance, were retained and displayed in Table 13, in the Appendix. Most of the items were saturated on the 1st (six items) and 2nd (four items) factor, while the 3rd, 4th and 5th factor each contain two items. Although the present solution does not clearly reflect the three-phase structure, it clearly divides PI and PIII, by clustering their items in separate factors. On the first factor, all the items from PI and most of the items from PII are saturated. 2nd and 3rd factor both contain items from PII and PIII. Finally, 4th and 5th factor contain only items from PIII. A full correlation matrix for further inspection of the evaluation is available in Table 15 .

\section{Discussion}

In this chapter we will relate the validation results to our expectations and previous findings, stated in the related work section. We will then discuss the validation results for each phase in the context of methodological limitations and we will provide suggestions for future work. Finally, we will indicate the implications of the PERSONA test and possibili- 
Table 6

Descriptive statistics of PERSONA-80

\begin{tabular}{|c|c|c|c|c|c|c|c|c|}
\hline & $\mathrm{N}$ & Mean & Std. Deviation & Coefficient of variance & \multicolumn{2}{|c|}{ Skewness } & \multicolumn{2}{|c|}{ Kurtosis } \\
\hline & Statistic & Statistic & Statistic & Statistic & Statistic & Std. Error & Statistic & Std. Error \\
\hline Needs & 83.00 & 38.08 & 7.38 & 0.19 & -0.97 & 0.26 & 1.43 & 0.52 \\
\hline Cognitive schemas & 83.00 & 162.65 & 20.45 & 0.13 & 0.16 & 0.26 & -0.48 & 0.52 \\
\hline Coping strategy & 83.00 & 169.29 & 17.02 & 0.10 & -0.32 & 0.26 & 0.29 & 0.52 \\
\hline
\end{tabular}

Table 7

Results of normality tests for PERSONA-80

\begin{tabular}{lllllll}
\hline & \multicolumn{3}{c}{ Kolmogorov-Smirnov } & \multicolumn{3}{c}{ Shapiro-Wilk } \\
\hline \multicolumn{1}{c}{ Phase } & Statistic & df & Sig. & Statistic & df & Sig. \\
Needs & 0.10 & 83.00 & 0.05 & 0.94 & 83.00 & 0.00 \\
Cognitive schemas & 0.06 & 83.00 & $.200^{*}$ & 0.98 & 83.00 & 0.37 \\
Coping strategy & 0.09 & 83.00 & 0.17 & 0.98 & 83.00 & 0.10 \\
*. This is a lower bound of the true significance. & & &
\end{tabular}

Table 8

\begin{tabular}{llll}
\multicolumn{4}{l}{ Results of reliability analysis for PERSONA-80 } \\
\hline Scale & Cron.'s Alpha & Cron.'s Alpha (Std. Items) & N Items \\
\hline Needs & 0.732 & 0.736 & 8 \\
Cognitive schemas & 0.875 & 0.881 & 32 \\
Coping strategy & 0.683 & 0.679 & 40 \\
\hline
\end{tabular}

ties for its future use in occupational and clinical contexts.

Factor analysis of PERSONA-80 did not fully confirm the architecture, although it showed the divergence between the first and the third phase. Saturation of the largest portion of items on the first factor, equally distributed among all three-phases, indicates the existence of higher-order factor which should be examined on a larger sample. Distribution of items among the 2 nd and the 3rd factor implies a relationship between attributed importance and positivity of memories with certain coping tendencies, indicating thus partial connection between PHASE II and PHASE III. We can assume that importance of autobiographical memories can predict certain defense mechanisms, while positivity of memories can predict other defense mechanisms and that cognitive schemas in general predict stress-responses of the individual. Finally, absence of items from the PHASE I on the 2nd and the 3rd factor shows that PHASE I and PHASE III measure different constructs.

Leptokurtic distribution of the PHASE I implies poor sen-

Table 9

Dimensions of PERSONA-16 divided by phase, identified as the highest contributors for predictive validity from the PERSONA-80 pool, obtained via Greedystepwise and Cfs SubsetEval analysis

\begin{tabular}{ccc}
\hline PI & PII & PIII \\
\hline PI.EC.P & PII.SP.IS.P & PIII.IB.2 \\
PI.IC.P & PII.SP.IC.P & PIII.IR.2 \\
& PII.SP.EC.P & PIII.IM.3 \\
& PII.OP.EC.O & PIII.RD.11 \\
& PII.OP.EC.P & PIII.RD.5 \\
& PII.OP.IS.P & PIII.DA.1 \\
& PII.SI.IC.O & PIII.DA.5 \\
\hline
\end{tabular}

sitivity of this scale, which can be justified by the small number of items. Moderate positive skew in the distribution indicates possible socially desirable answering of the participants which can be improved in future work by reversing part of the items. The two-factor solution of the first phase, which divides needs in cognitive and sensorial, implies differences in the way of processing the stimuli among individuals, which could explain differences in coping responses among individuals and certain distortions in cognitive schemas. However, in future work and with more data points, internal and external facets of those needs should be explored, in order to explore how they relate to self- and world- schema and to inner- and outer-oriented coping strategies.

PHASE II showed very good internal consistency and a clear two-factor solution, identifying subscales for Positivity and Importance, which partially supports construction the hypothesis of PHASE II in which we wanted to assess positive affect and relevance attributed to certain autobiographical memories of an individual. This enables inferences about overstimulated an understimulated areas of his/her life and how they reflect on coping maturity. Nevertheless, with the increase of samples it is important to individuate subscales for evaluating separately self-schema and world-schema memories, in order to relate them to inner- and outer- oriented coping strategies from the third phase.

PHASE III is the most complex scale for validation in this test, because it measures e heterogeneous set of responses, on different levels (emotional, cognitive and behavioral). It is also the most experimental scale because responses are being measured on a maturity continuum, where maturity is here defined as the extent of distortion of cognitive schemas. Since it measures heterogeneous responses, which are context dependent and thus less reliable by nature, it consequently shows weaker reliability than previous phases, i.e. lower internal consistency. With regards to factor structure, evaluation shows a fairly clear division on inneroriented and outer-oriented defense mechanisms. Herein, inner-oriented mechanisms refer to employing inner psychological resources for reducing anxiety coming from stressful stimuli. Meanwhile, outer-oriented mechanisms refer to expressing anxiety to the outer world when perceiving stressful experiences. This division offers an interesting view on the classification of defense mechanisms which allows to predict the tendency of an individual to show violent and autodestructive behaviors in stressful situations. Furthermore, this allows us to relate the coping orientation to internaliz- 
Table 10

Descriptive statistics of PERSONA-16

\begin{tabular}{|c|c|c|c|c|c|c|c|c|}
\hline & $\mathrm{N}$ & Mean & Std. Deviation & Coefficient of variance & \multicolumn{2}{|c|}{ Skewness } & \multicolumn{2}{|c|}{ Kurtosis } \\
\hline & Statistic & Statistic & Statistic & Statistic & Statistic & Std. Error & Statistic & Std. Error \\
\hline PERSONA 16 & 83.00 & 78.16 & 10.29 & 0.13 & -1.20 & 0.26 & 1.47 & 0.52 \\
\hline
\end{tabular}

Table 11

Results of normality tests for PERSONA-16

\begin{tabular}{llllll}
\hline \multicolumn{2}{l}{ Kolmogorov-Smirnov } & \multicolumn{3}{c}{ Shapiro-Wilk } \\
\hline Statistic & df & Sig. & Statistic & df & Sig. \\
0.12 & 83 & 0.01 & 0.9 & 83 & 0 \\
\hline
\end{tabular}

Table 12

Results of reliability analysis for PERSONA 16, showing values of Cronbach Alpha for internal consistency

\begin{tabular}{ccc}
\hline Cron.'s Alpha & Cron.'s Alpha Based on Std. Items & N Items \\
\hline 0.714 & 0.724 & 16 \\
\hline
\end{tabular}

ing and externalizing symptoms of psychopathological disorders. Finally, this structure can give us a valuable insight on formation and handling of stress because it enables to relate inner- an outer- oriented mechanisms to self- and worldschema or with emotional and rational processing of social information in future work.

The purified version of the PERSONA-80 contains 16 items identified as the strongest predictors of the presence of psychiatric diagnosis, thus named PERSONA-16. The purified test maintains strong internal consistency, showing that items are sufficiently intercorrelated and that they sufficiently correlate with the total score. This aligns with the fact that they predict the same condition, i.e. a generic psychopathological condition. Finally, PERSONA-16 divided PHASE I and PHASE III on different factors, providing clearer factor structure than PERSONA-80. Although this did not justify the use of a three-phase model, it indicated the presence of two general factors, implying once again that hierarchical factor analysis would be more appropriate statistical method for validation of this model. Furthermore, this scale was constructed for the purposes of predictive validity, thus lacks in construct validity are acceptable.

Overall, the purified version, the PERSONA-16 test, fulfilled its purpose by showing very good predictive capacity of the generic psychopathological condition. It exhibited items of all three-phases; need for stimuli, cognitive schemas and defense mechanisms; all three are strong predictors for psychopathological disorders. Both the original 80-item instrument and the purified 16-item test showed good internal consistency, despite the small evaluation sample and the fact that general and clinical sampling were done in two different languages (English and Italian respectively). We suggest to further explore relationships among factors in future work by means of hierarchical factor analysis, in order to define general and specific factors of the model. Finally, we should take into account that validation has been conducted on a small sample, potentially unbalanced by age, which can be improved within the next validations.

This three-phase architecture provides both theoretical and practical implications. From the theoretical point of view, the three-phase architecture offers a novel perspective on stress-coping mechanisms: rather than measuring a set of behavioral responses to stress, it measures how the individual receives and elaborates stimuli, stores them into his/her mental state and produces behavior out of a mental state. By relating stimuli-optimality to cognitive schemas and cognitive schemas to defense behavior responses, we enable a better understanding of the development of maladaptive mental and behavioral patterns of an individual. From the practical perspective, it offers a novel method of classification which is based on latent capability of a person to translate mental state into mature and constructive behavior. PERSONA test can find its application in occupational settings, when evaluating candidates in high-stress professional roles, such as soldiers, pilots or surgeons, as well as in clinical settings, where it can assess mental strengths and weaknesses of the patient in confronting stress-related illnesses and to monitor his/her recovery.

\section{Conclusion}

Validation analysis of PERSONA-80, conducted on the sample of individuals from general and clinical population, confirmed good psychometric properties of the instrument. Firstly, it showed adequate levels of internal consistency both at a test-level and at a scale-level. Factor structure did not fully confirm the three-phase architecture, but it sufficiently divided PHASE I from PHASE III, therefore justifying the separation rationale. Secondly, by extracting the items with the highest predictive capability toward classifying the clinical psychopathological condition, PERSONA-16 was created, thus fulfilling the practical objective of PERSONA test. PERSONA-16 maintained good internal consistency and provided a clear factor structure. While within this validation scope we showed the predictive validity towards detecting generic psychopathological conditions, the well-investigated theoretical foundations of the test can lead to further classification potential, with a focus on analyzing stress formation, handling and manifestation, useful for occupational or military use, or whenever the tested individual is potentially subject to a high stress task. We highlight the importance of the introduced three-phase model and its transactional nature, which can be use to the scientific community as underlying structure to multiple test implementations. We hereby pro- 
vide at the end of this text the full PERSONA-16 instrument to encourage its widespread validation and use in multiple contexts (Table 17 for the English version and Table 18 for the Italian version).

\section{References}

Andrews, G., Pollock, C., \& Stewart, G. (1989). The determination of defense style by questionnaire. Archives of general psychiatry, 46(5), 455-460.

Andrews, G., Singh, M., \& Bond, M. (1993). The defense style questionnaire. The Journal of nervous and mental disease, 181(4), 246-256.

Arbanasić, K., \& Kirk, N. H. (2017). Understanding resilience by relating stimuli optimality to schema constructs. In Proceedings of the 59th annual conference of the international military testing association, bern, switzerland.

Bond, M. (2004). Empirical studies of defense style: relationships with psychopathology and change. Harvard review of psychiatry, 12(5), 263-278.

Bond, M., Paris, J., \& Zweig-Frank, H. (1994). Defense styles and borderline personality disorder. Journal of Personality Disorders, 8(1), 28-31.

Carver, C. S., Scheier, M. F., \& Weintraub, J. K. (1989). Assessing coping strategies: a theoretically based approach. Journal of personality and social psychology, 56(2), 267.

Cattell, R. B. (1966). The scree test for the number of factors. Multivariate behavioral research, 1(2), 245-276.

Cramer, P. (1998). Coping and defense mechanisms: What's the difference? Journal of Personality, 66(6), 919-946.

Dekel, R., Solomon, Z., Elklit, A., \& Ginzburg, K. (2004). World assumptions and combat-related posttraumatic stress disorder. The Journal of social psychology, 144(4), 407-420.

Endler, N. S., Parker, J. D., de Ridder, D. T., \& van Heck, G. L. (2004). Ciss: Coping inventory for stressful situations. Harcourt.

Fowler, D., Freeman, D., Smith, B., Kuipers, E., Bebbington, P., Bashforth, H., ... others (2006). The brief core schema scales (bcss): psychometric properties and associations with paranoia and grandiosity in non-clinical and psychosis samples. Psychological medicine, 36(6), 749-759.

Hebb, D. O. (1955). Drives and the cns (conceptual nervous system). Psychological review, 62(4), 243.

Janoff-Bulman, R. (1989). Assumptive worlds and the stress of traumatic events: Applications of the schema construct. Social cognition, 7(2), 113-136.

Kaiser, H. F. (1960). The application of electronic computers to factor analysis. Educational and psychological measurement, 20(1), 141-151.

Kirk, N. H., \& Arbanasić, K. (2017a, September). An assessment method for monitoring reliability of individuals in high risk professions, via stress defense maturity analysis and artificial intelligence. (IT Patent App. 102,017,000,108,892)

Kirk, N. H., \& Arbanasić, K. (2017b). Reducing inoperative days of soldiers under stress: A novel approach. In Proceedings of the 59th annual conference of the international military testing association, bern, switzerland.
Leuba, C. (1955). Toward some integration of learning theories: The concept of optimal stimulation. Psychological Reports, 1(1), 27-33.

Lishman, W. A. (1974). The speed of recall of pleasant and unpleasant experiences. Psychological Medicine, 4(2), 212-218.

Mayo, P. R. (1983). Personality traits and the retrieval of positive and negative memories. Personality and Individual Differences, $4(5), 465-471$.

Nunally, J., \& Bernstein, I. (1994). Psychometric theory. vol. McGraw-Hill.

Pearson, P. H. (1970). Relationships between global and specified measures of novelty seeking. Journal of Consulting and Clinical Psychology, 34(2), 199.

Pearson, P. H. (1971). Differential relationships of four forms of novelty experiencing. Journal of Consulting and Clinical Psychology, 37(1), 23.

Pelham, B. W., \& Swann, W. B. (1989). From self-conceptions to self-worth: on the sources and structure of global self-esteem. Journal of personality and social psychology, 57(4), 672.

Raju, P. S. (1980). Optimum stimulation level: Its relationship to personality, demographics, and exploratory behavior. Journal of consumer research, 7(3), 272-282.

Steenkamp, J.-B. E., \& Baumgartner, H. (1992). The role of optimum stimulation level in exploratory consumer behavior. Journal of consumer research, 19(3), 434-448.

Stotland, E., \& Pendleton, M. (1989). Workload, stress, and strain among police officers. Behavioral Medicine, 15(1), 5-17.

Vaillant, G. E. (1994). Ego mechanisms of defense and personality psychopathology. Journal of abnormal psychology, 103(1), 44.

Vaillant, G. E., Bond, M., \& Vaillant, C. O. (1986). An empirically validated hierarchy of defense mechanisms. Archives of General Psychiatry, 43(8), 786-794.

Zuckerman, M. (1994). Behavioral expressions and biosocial bases of sensation seeking. Cambridge university press.

\section{Acknowledgements}

We gratefully acknowledge the funding provided by WestTech Ventures GmbH to Cambridge Humanae ltd., which enabled the research \& development of the presented system, as well as the extensive search of customers and partners. We are grateful to Rebecca Ciacchini for having administered the test in the clinical context. A further acknowledgement goes to Andrea Stefani for having reviewed an intermediate version of this paper.

\section{Appendix}


Table 13

Results of the Exploratory Factor Analysis (EFA) for PERSONA-16, identifying 5 independent factors

\begin{tabular}{llllll}
\hline \multicolumn{7}{c}{ Items } & \multicolumn{6}{c}{ Component } \\
\hline PII.SP.EC.P & $\mathbf{0 . 8 0}$ & -0.07 & -0.22 & -0.35 & -0.31 \\
PI.EC.P & $\mathbf{0 . 7 5}$ & 0.14 & -0.35 & -0.18 & -0.28 \\
PII.OP.EC.P & $\mathbf{0 . 7 1}$ & -0.03 & -0.18 & -0.28 & -0.25 \\
PII.OP.EC.O & $\mathbf{0 . 6 5}$ & -0.35 & -0.40 & -0.42 & -0.04 \\
PI.IC.P & $\mathbf{0 . 6 3}$ & -0.26 & -0.17 & -0.52 & -0.45 \\
PII.OP.IS.P & $\mathbf{0 . 6 0}$ & -0.11 & -0.11 & 0.19 & -0.33 \\
PIII.DA.5 & 0.14 & $\mathbf{0 . 6 5}$ & -0.04 & -0.30 & -0.18 \\
PII.SI.IC.O & 0.29 & $\mathbf{- 0 . 6 4}$ & 0.11 & -0.08 & -0.40 \\
PII.SP.IS.P & 0.47 & $\mathbf{- 0 . 5 3}$ & -0.37 & -0.44 & -0.23 \\
PIII.RD.5 & 0.50 & $\mathbf{0 . 5 3}$ & 0.04 & -0.18 & -0.43 \\
PII.SP.IC.P & 0.31 & 0.14 & $\mathbf{- 0 . 8 1}$ & -0.04 & -0.13 \\
PIII.IM.3 & -0.26 & 0.23 & $\mathbf{0 . 7 8}$ & 0.29 & 0.22 \\
PIII.IR.2 & 0.38 & 0.16 & -0.03 & $\mathbf{- 0 . 7 7}$ & -0.06 \\
PIII.RD.11 & 0.16 & 0.03 & -0.25 & $\mathbf{- 0 . 7 5}$ & -0.32 \\
PIII.IB.2 & 0.35 & -0.04 & -0.04 & -0.29 & $\mathbf{- 0 . 7 9}$ \\
PIII.DA.1 & -0.25 & -0.06 & 0.43 & 0.01 & $\mathbf{0 . 6 9}$ \\
\hline **. Correlation is significant at the 0.01 level (2-tailed). \\
*. Correlation is significant at the 0.05 level (2-tailed).
\end{tabular}

Table 14

Results of the Confirmatory Factor Analysis (CFA) for PERSONA-80, displaying 3-factor solution

\begin{tabular}{|c|c|c|c|c|c|c|c|}
\hline \multicolumn{5}{|c|}{ Component } & \multicolumn{3}{|c|}{ Component } \\
\hline Items & $\mathbf{1}$ & 2 & 3 & Items & 1 & 2 & 3 \\
\hline PII.SP.IC.O & 0.74 & -0.10 & -0.31 & PII.OI.IC.P & 0.24 & 0.59 & -0.14 \\
\hline PII.SP.IS.O & 0.73 & 0.06 & -0.12 & PII.OI.ES.P & 0.13 & 0.57 & -0.13 \\
\hline PI.EC.P & 0.70 & 0.06 & -0.22 & PII.SI.IC.O & 0.20 & 0.56 & -0.17 \\
\hline PII.SP.EC.P & 0.68 & 0.14 & -0.34 & PII.OI.IS.P & 0.34 & 0.55 & -0.22 \\
\hline PII.OP.IC.P & 0.67 & 0.06 & -0.42 & PII.SI.IC.P & 0.13 & 0.55 & 0.42 \\
\hline PII.OP.EC.P & 0.63 & 0.02 & -0.27 & PII.SI.IS.P & 0.21 & 0.54 & 0.15 \\
\hline PII.OI.EC.O & 0.61 & 0.12 & -0.08 & PII.OI.IS.O & 0.08 & 0.54 & 0.19 \\
\hline PI.IS.P & 0.59 & -0.09 & 0.24 & PIII.DA.5 & 0.27 & -0.51 & 0.01 \\
\hline PII.OP.IS.P & 0.57 & 0.16 & -0.16 & PIII.RD.15 & 0.24 & -0.45 & -0.37 \\
\hline PI.IC.P & 0.57 & 0.17 & -0.41 & PIII.RD.6 & 0.36 & -0.45 & 0.11 \\
\hline PIII.RD.4 & 0.56 & -0.05 & -0.01 & PII.SI.EC.O & -0.11 & 0.42 & -0.15 \\
\hline PI.EC.O & 0.54 & 0.00 & -0.09 & PI.IC.O & 0.30 & 0.39 & -0.26 \\
\hline PIII.IB.5 & 0.54 & -0.18 & -0.07 & PIII.RD.8 & -0.21 & 0.35 & 0.10 \\
\hline PIII.RD.5 & 0.53 & -0.22 & -0.03 & PIII.RD.10 & 0.25 & -0.31 & -0.29 \\
\hline PI.IS.O & 0.53 & 0.08 & 0.02 & PIII.IR.3 & 0.11 & -0.27 & 0.17 \\
\hline PI.ES.O & 0.52 & -0.06 & -0.20 & PIII.DA.4 & -0.10 & 0.23 & 0.23 \\
\hline PIII.IB.2 & 0.49 & 0.02 & -0.27 & PIII.RD.12 & 0.08 & -0.21 & 0.05 \\
\hline PIII.IR.4 & 0.48 & 0.06 & -0.44 & PIII.IR.1 & 0.04 & 0.20 & 0.00 \\
\hline PII.SP.ES.O & 0.48 & -0.03 & -0.22 & PIII.IM.3 & -0.20 & -0.17 & 0.70 \\
\hline PII.OI.IC.O & 0.47 & 0.30 & -0.10 & PII.SP.ES.P & 0.17 & 0.24 & -0.69 \\
\hline PII.OP.ES.O & 0.45 & -0.04 & -0.32 & PIII.RD.13 & -0.15 & -0.04 & 0.60 \\
\hline PIII.IR.2 & 0.45 & -0.32 & -0.17 & PII.SP.IC.P & 0.31 & -0.13 & -0.57 \\
\hline PIII.RD.7 & -0.43 & 0.25 & 0.28 & PII.SP.IS.P & 0.32 & 0.24 & -0.56 \\
\hline PIII.RD.9 & 0.42 & 0.24 & 0.02 & PII.OP.EC.O & 0.47 & 0.20 & -0.53 \\
\hline PIII.IR.5 & 0.41 & -0.34 & -0.36 & PIII.RD.1 & -0.13 & -0.03 & 0.52 \\
\hline PII.OP.IS.O & 0.40 & -0.02 & -0.26 & PIII.IA.3 & 0.04 & 0.01 & 0.52 \\
\hline PII.SI.EC.P & 0.38 & 0.30 & -0.04 & PII.SP.EC.O & 0.32 & -0.11 & -0.52 \\
\hline PII.OI.ES.O & 0.37 & 0.23 & 0.27 & PIII.IA.1 & -0.12 & 0.24 & 0.51 \\
\hline PIII.IM.5 & 0.37 & -0.09 & 0.30 & PIII.DA.2 & 0.11 & 0.06 & 0.48 \\
\hline PII.SI.ES.O & 0.36 & 0.25 & 0.28 & PIII.RD.2 & -0.11 & -0.38 & 0.47 \\
\hline PI.ES.P & 0.36 & -0.23 & 0.19 & PII.OP.IC.O & 0.32 & 0.17 & -0.42 \\
\hline PII.OI.EC.P & 0.35 & 0.22 & -0.20 & PIII.DA.1 & -0.28 & -0.03 & 0.39 \\
\hline PIII.DA.3 & 0.34 & -0.20 & -0.03 & PIII.RD.11 & 0.32 & -0.19 & -0.33 \\
\hline PIII.IM.4 & 0.29 & -0.06 & 0.09 & PIII.IM.1 & -0.15 & 0.15 & 0.31 \\
\hline PIII.IM.2 & 0.29 & -0.06 & 0.00 & PIII.IB.1 & 0.14 & -0.27 & -0.27 \\
\hline PII.OP.ES.P & 0.25 & 0.14 & -0.15 & PIII.IB.3 & -0.22 & -0.09 & 0.27 \\
\hline PIII.RD.3 & 0.24 & 0.03 & 0.02 & PIII.IA.5 & 0.12 & -0.08 & 0.27 \\
\hline PIII.RD.14 & 0.23 & 0.08 & -0.05 & PIII.IB.4 & -0.19 & 0.10 & 0.26 \\
\hline PII.SI.IS.O & -0.02 & 0.63 & 0.01 & PIII.IA.4 & 0.14 & 0.04 & 0.23 \\
\hline PII.SI.ES.P & 0.15 & 0.62 & -0.05 & PIII.IA.2 & 0.00 & -0.08 & 0.23 \\
\hline
\end{tabular}



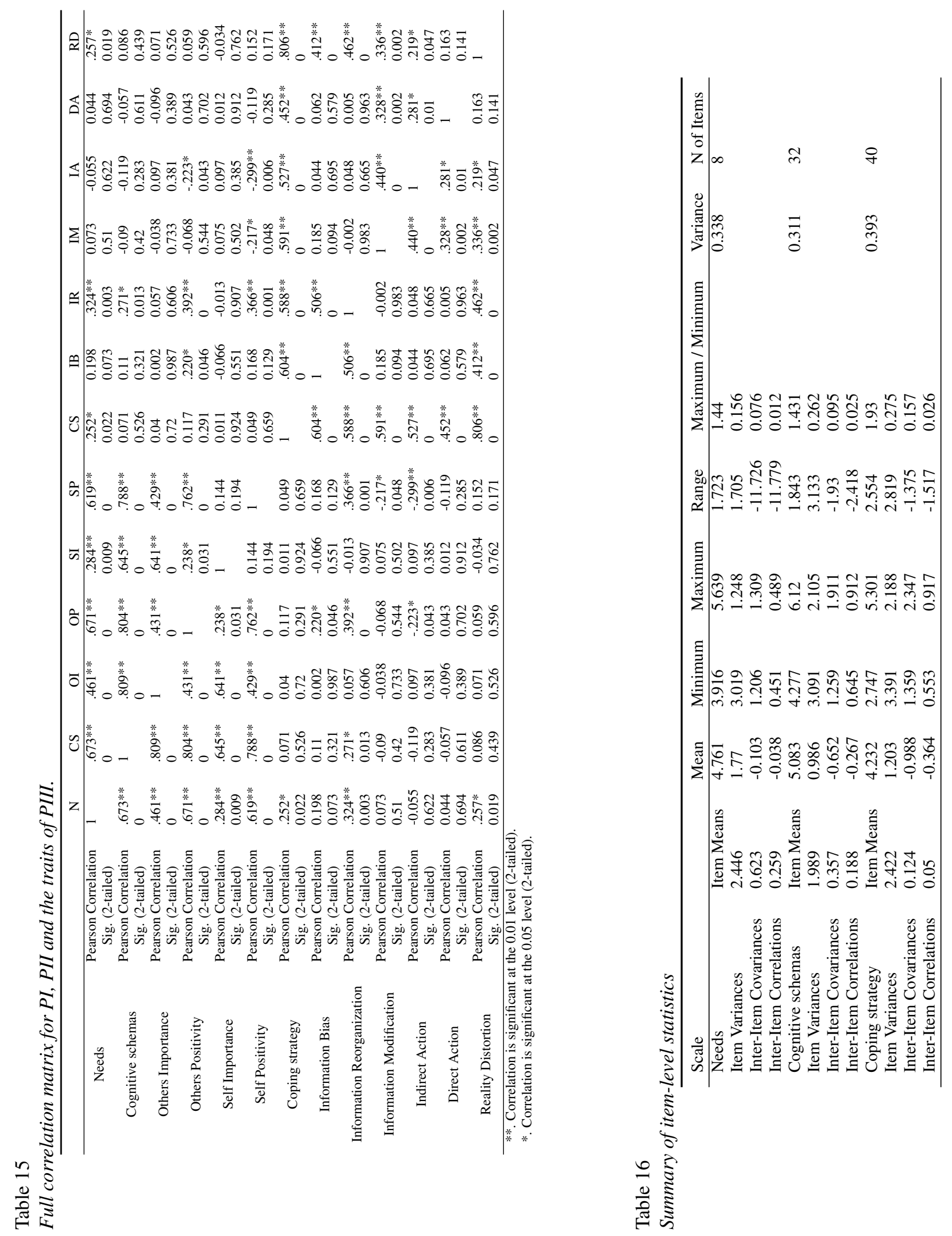
PERSONA: A PSYCHOMETRIC MONITORING INSTRUMENT FOR HIGH-STRESS INDIVIDUALS

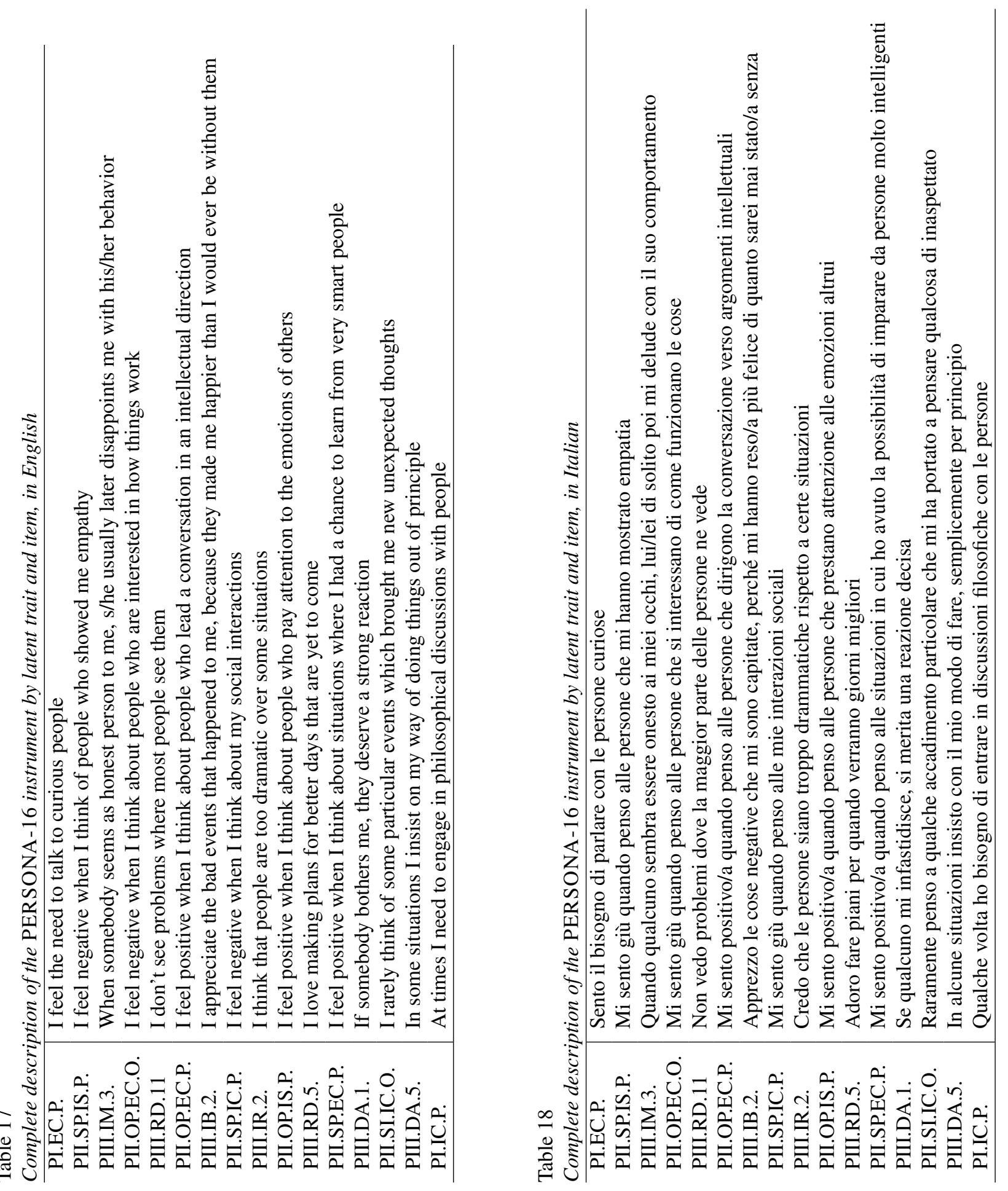

